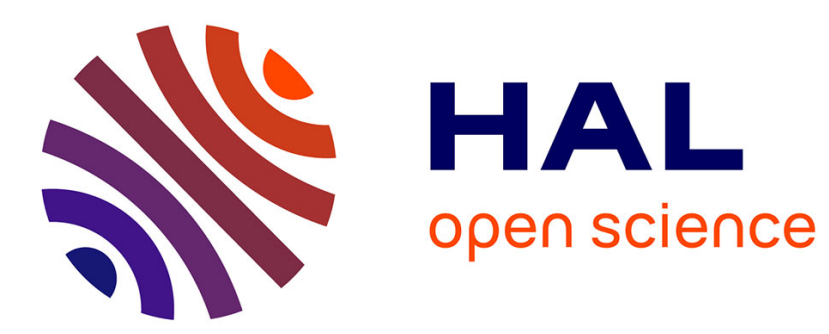

\title{
Hacheur de faisceau utilisant la recirculation de la puissance hyperfréquence
}

\author{
M. Boussoukaya, A. Septier, L. Wartski
}

\section{To cite this version:}

M. Boussoukaya, A. Septier, L. Wartski. Hacheur de faisceau utilisant la recirculation de la puissance hyperfréquence. Revue de Physique Appliquée, 1976, 11 (2), pp.299-303. 10.1051/rphysap:01976001102029900 . jpa-00244061

\section{HAL Id: jpa-00244061 https://hal.science/jpa-00244061}

Submitted on 1 Jan 1976

HAL is a multi-disciplinary open access archive for the deposit and dissemination of scientific research documents, whether they are published or not. The documents may come from teaching and research institutions in France or abroad, or from public or private research centers.
L'archive ouverte pluridisciplinaire HAL, est destinée au dépôt et à la diffusion de documents scientifiques de niveau recherche, publiés ou non, émanant des établissements d'enseignement et de recherche français ou étrangers, des laboratoires publics ou privés. 


\title{
HACHEUR DE FAISCEAU UTILISANT LA RECIRCULATION DE LA PUISSANCE HYPERFRÉQUENCE
}

\author{
M. BOUSSOUKAYA, A. SEPTIER et L. WARTSKI \\ Institut d'Electronique Fondamentale $\left({ }^{*}\right)$, \\ Université Paris-Sud, Bâtiment 220, 91405 Orsay, France
}

(Reçu le 11 juillet 1975, accepté le 27 octobre 1975)

\begin{abstract}
Résumé. - Un hacheur de faisceau utilisant une ligne à méandres placée dans une boucle de recirculation de la puissance HF a été étudié théoriquement et expérimentalement. L'anneau résonnant en onde stationnaire permet d'obtenir de meilleurs résultats que celui fonctionnant en onde progressive. Le gain de puissance obtenu avec l'anneau résonnant en onde stationnaire est $\simeq 9$. Il permet d'obtenir des paquets d'électrons de très courte durée $(\simeq 10 \mathrm{ps})$ avec une puissance HF de quelques watts en défléchissant des faisceaux de $30 \mathrm{keV}$ d'énergie et de $1 \mathrm{~mA}$ d'intensité.
\end{abstract}

Abstract. - An electron beam chopper using a meander line has been studied. The RF power is injected through a resonant feedback loop either in a stationary wave or traveling wave arrangement. In the former case, with which better results are obtained, the power build up ratio is $\simeq 9$. Experiments show the possibility of obtaining short duration bunches $(\simeq 10 \mathrm{ps})$ with $\mathrm{RF}$ power of the order of a few watts by the deflection of $30 \mathrm{keV}, 1 \mathrm{~mA}$ electron beams.

1. Introduction. - La production continue de paquets d'électrons de courte durée ( 1 à $10 \mathrm{ps}$ ) en vue de leur injection dans un accélérateur linéaire supraconducteur [1] peut être réalisée à l'aide d'un groupeur de type conventionnel (buncher) ou bien d'un hacheur de faisceau, lorsque les intensités de courant mises en jeu sont faibles.

Le hacheur de faisceau que nous avons mis au point se compose d'un déflecteur HF qui impose au faisceau injecté sur l'axe une déviation suivant deux directions orthogonales. A une distance $D$ du déflecteur, on place alors sur l'axe du système un collecteur plan percé d'un trou de faible diamètre que le faisceau traverse pendant une très courte partie de la période, ce qui fournit, en arrière du diaphragme, des paquets d'électrons de très faible durée à la fréquence de fonctionnement du système ( 1 à $3 \mathrm{GHz}$ dans nos expériences).

Par raison de commodité, les dispositifs d'injection fonctionnent à la température ambiante; dans ces conditions, les groupeurs à cavité résonnante cylindrique nécessitent des puissances $\mathrm{HF}$ relativement importantes (plusieurs $\mathrm{kW}$ ). Au contraire, dans les différents systèmes déflecteurs hyperfréquences utilisés [2, 3, 4] (cavités cylindriques lisses ou lignes à méandres), la puissance HF nécessaire à l'excitation du champ déflecteur est beaucoup plus faible (quelques dizaines de watts au maximum).

(*) Laboratoire associé au C. N. R. S.
La largeur des paquets est fonction de la puissance HF injectée, ainsi que de la longueur du bras de levier optique qui suit le déflecteur. Nous avons étudié théoriquement et expérimentalement cette possibilité d'obtenir des paquets d'électrons de très faible durée, en mettant en œuvre la recirculation de la puissance HF dans un anneau résonnant renfermant la ligne déflectrice, dans le but de réduire au maximum la puissance HF nécessaire.

2. Anneaux résonnants. - Notre déflecteur utilise une ligne à méandres (Fig. 1), structure à très large bande par excellence, qui est souvent employée dans les oscilloscopes haute fréquence. Pour un tel fonctionnement, la ligne alimentée en ondes progressives, est fermée sur son impédance caractéristique.

Par contre, dans un fonctionnement à fréquence fixe, la puissance perdue dans la charge adaptée terminant le dispositif peut être réutilisée en plaçant la ligne dans une boucle de recirculation de longueur électrique convenable, qui constitue alors un anneau résonnant.

La recirculation de la puissance HF dans un anneau a été étudiée et utilisée en pratique dans le cas des accélérateurs linéaires $[5,6]$. Toutefois, l'étude théorique d'un tel système n'a été faite que pour le cas du régime à onde progressive.

Dans notre étude, nous nous sommes intéressés à l'utilisation de la recirculation de la puissance dans le cas des déflecteurs HF, aussi bien en régime d'onde progressive qu'en régime d'onde stationnaire. 

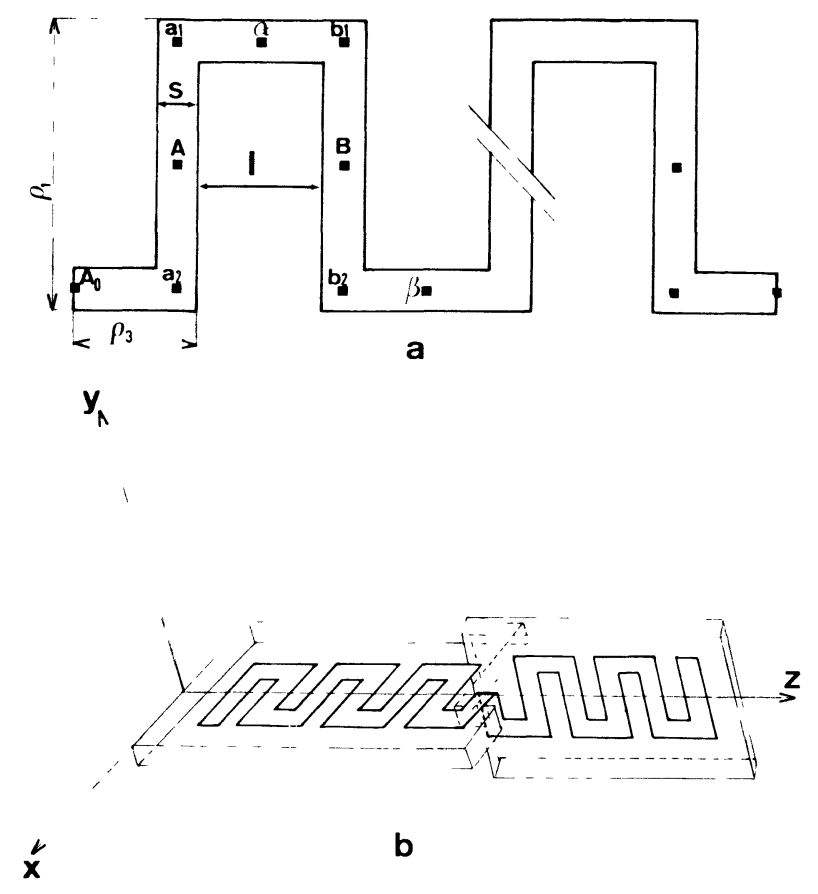

FIG. 1. - Double déflecteur à méandres à ruban unique.

3. Théorie de l'anneau résonnant. - 3.1 ANNEAU RÉSONNANT EN ONDES PROGRESSIVES. - Le couplage entre le générateur $\mathrm{HF}$ et l'anneau s'effectue au travers d'un coupleur directif.

On utilise la relation matricielle existant entre les
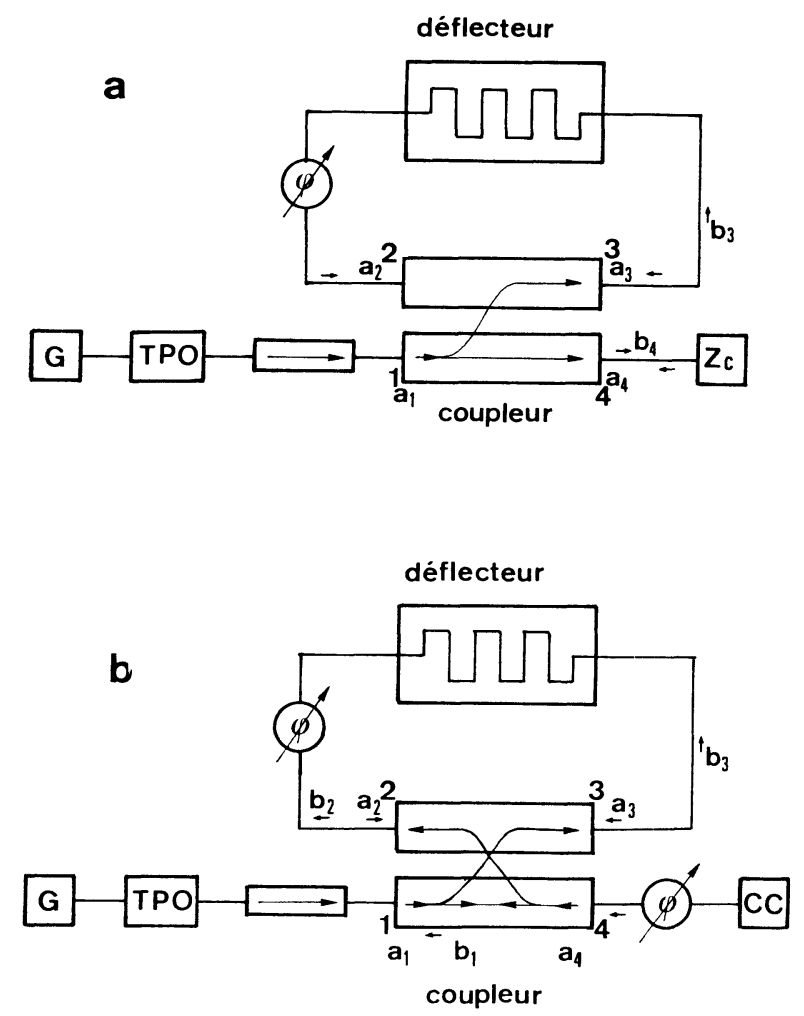

Fig. 2. - Anneaux de recirculation de la puissance HF résonnants : $a$ ) en ondes progressives ; $b$ ) en ondes stationnaires. amplitudes des ondes incidentes $a_{j}$ et des ondes réfléchies $b_{i}$ dans les différentes voies de ce coupleur (Fig. 2)

$$
\left(b_{i}\right)=\left(S_{i, j}\right)\left(a_{j}\right)
$$

où $S_{i j}$ représente la matrice de distribution du système, dont les éléments désignent d'une part les coefficients de transmission entre les voies $i$ et $j$ du coupleur lorsque $i \neq j$, d'autre part les coefficients de réflexion dans la voie $i$ lorsque $i=j$.

En tenant compte des propriétés propres du coupleur telles que la symétrie, l'absence de pertes dans le coupleur et l'adaptation d'impédance sur l'une des voies, on peut trouver les expressions des amplitudes $b_{i}$ dans les quatre voies du coupleur bidirectionnel. Dans le cas d'un anneau résonnant en onde progressive (Fig. 2a), on trouve :

$$
\begin{aligned}
& b_{1}=0 \\
& b_{2}=0 \\
& b_{3}= \pm j \alpha a_{1}+\gamma \beta_{\mathrm{e}} b_{3} \mathrm{e}^{j \varphi} \\
& b_{4}=a_{1} \pm j \alpha \gamma b_{3} \mathrm{e}^{j \varphi}
\end{aligned}
$$

On a posé

$$
\beta=\frac{\sqrt{C_{0}-1}}{\sqrt{C_{0}}} \text { et } \alpha=\frac{1}{\sqrt{C_{0}}}
$$

$C_{0}$ représente le coefficient de couplage du coupleur directif, $\gamma$ l'atténuation dans l'anneau et $\varphi$ le déphasage total à travers l'anneau de recirculation.

Exprimées en fonction de l'onde incidente $a_{1}$, les amplitudes des ondes $b_{3}$ (circulant dans la boucle) et $b_{4}$ (allant vers la charge adaptée), sont :

$$
\begin{aligned}
& \left|b_{3}\right|=\sqrt{b_{3} b_{3}^{*}}=\frac{a_{1}}{\sqrt{C_{0}}-\gamma \sqrt{C_{0}-1} \mathrm{e}^{j \varphi}} \\
& \left|b_{4}\right|=\sqrt{b_{4} b_{4}^{*}}=\frac{\left(\sqrt{C_{0}-1}-\gamma \sqrt{C_{0}} \mathrm{e}^{j \varphi}\right) a_{1}}{\sqrt{C_{0}}-\gamma \sqrt{C_{0}-1} \mathrm{e}^{j \varphi}} .
\end{aligned}
$$

L'amplitude $\left|b_{3}\right|$ est maximale lorsque $\varphi=0$.

Le maximum d'amplitude de l'onde circulant dans la boucle en fonction du couplage est obtenu lorsque

$$
\frac{\mathrm{d}}{\mathrm{d} C_{0}}\left|b_{3}\right|=0
$$

soit

$$
C_{0}=\frac{1}{1-\gamma}
$$

En remplaçant $C_{0}$ par son expression (6) dans la formule (4), on trouve :

$$
b_{4}=0 \text {. }
$$

Ce résultat représente la condition optimale du régime permanent dans la boucle.

Dans cet anneau résonnant en onde progressive, l'amplitude du champ électrique déflecteur, exprimée en fonction de l'amplitude du champ incident, du coeffi- 
cient de couplage et des pertes totales dans la boucle, est donnée par :

$$
E_{1}=\frac{a_{1}}{\sqrt{C_{0}}-\gamma \sqrt{C_{0}-1}}
$$

3.2 ANNEAU RÉSONNANT EN ONDES STATIONNAIRES. - En remplaçant la charge résistive adaptée placée dans la voie $n^{0} 4$ par une impédance réactive formée par un déphaseur suivi d'un court-circuit, on peut réaliser un anneau à ondes stationnaires (Fig. 2b).

La matrice $S$ est définie uniquement par les propriétés du coupleur. En utilisant le même coupleur directif que précédemment, les amplitudes $b_{j}$ et $a_{i}$ se calculent dans ce cas grâce à l'expression matricielle suivante :

$\left[\begin{array}{l}b_{1} \\ b_{2} \\ b_{3} \\ b_{4}\end{array}\right]=\left[\begin{array}{cccc}0 & 0 & \pm j \alpha & \\ 0 & 0 & \beta & \pm j \alpha \\ \pm j \alpha & \beta & 0 & 0 \\ \beta & \pm j \alpha & 0 & 0\end{array}\right]\left[\begin{array}{l}a_{1} \\ \gamma b_{3} \mathrm{e}^{j \varphi} \\ \gamma b_{3} \mathrm{e}^{j \varphi_{2}} \\ a_{4}\end{array}\right]$

ce qui permet de trouver les expressions des amplitudes des ondes directe et rétrograde dans l'anneau :

$$
\begin{aligned}
& \left|b_{2}\right|=a_{4} /\left(\sqrt{C_{0}}-\gamma \sqrt{C_{0}-1}\right) \\
& \left|b_{3}\right|=a_{1} /\left(\sqrt{C_{0}}-\gamma \sqrt{C_{0}-1}\right) .
\end{aligned}
$$

Le champ déflecteur sera donné dans ce cas en un point de la ligne de cote $z$ par :

$$
E_{2}=\left|b_{2}\right| \cos \psi_{\mathrm{i}}(z, t)+\left|b_{3}\right| \cos \psi_{\mathrm{r}}(z, t)
$$

où $\psi_{\mathrm{i}}$ et $\psi_{\mathrm{r}}$ représentent respectivement les phases de l'onde directe et de l'onde rétrograde en ce point.

Lorsque les phases $\psi_{\mathrm{i}}$ et $\psi_{\mathrm{r}}$ sont convenablement ajustées, on obtient :

$$
E_{2}=\frac{a_{1}+a_{4}}{\sqrt{C_{0}}-\gamma \sqrt{C_{0}-1}} \cos \psi .
$$

L'amplitude du champ $E_{2}$ obtenu en régime d'onde stationnaire peut donc être supérieure à celle du champ $E_{1}$ de l'onde progressive, si $a_{4} \neq 0$.

4. Résultats expérimentaux. - L'anneau résonnant a été expérimenté d'abord dans les conditions schématisées sur la figure $2 a$ avec une charge adaptée sur la voie $4 \mathrm{du}$ coupleur directif (régime d'onde progressive) et ensuite dans les conditions de la figure $2 b$ avec un déphaseur suivi d'un court-circuit sur cette voie (régime d'onde stationnaire).

La puissance incidente a été mesurée à l'entrée de l'anneau résonnant (entre la ligne unidirectionnelle placée à la sortie du générateur de puissance et le coupleur directif) (Fig. 3).

Les puissances directe et rétrograde, circulant dans la boucle ont été mesurées en intercalant dans celle-ci un coupleur bidirectionnel de $20 \mathrm{~dB}$ (Fig. 3).

La précision de toutes les mesures est de $1 \%$ environ.

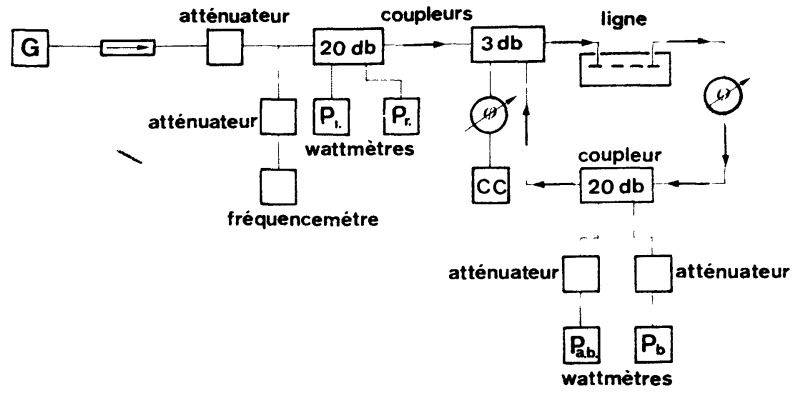

Fig. 3. - Dispositif expérimental permettant la mesure des puissances directe et rétrograde dans un anneau résonnant.

Les pertes d'insertion ont été estimées à $13 \%$ dans le cas d'une boucle courte.

Dans la pratique, l'anneau résonnant est placé dans une enceinte à vide et nécessite l'introduction de câbles de plusieurs mètres de longueur et de connexions supplémentaires, qui augmentent les pertes d'insertion (boucle longue).

Les résultats expérimentaux concernant le renforcement de la puissance circulant dans la boucle dans le sens direct, qu'on peut définir par le rapport :

$$
\alpha_{p}=\frac{\text { puissance directe }}{\text { puissance incidente }}
$$

sont représentés sur la figure 4 en fonction de la fréquence de fonctionnement $v$ et du coupleur utilisé.

Dans toutes les expériences effectuées sur les anneaux résonnants en régime d'onde progressive et d'onde sta-

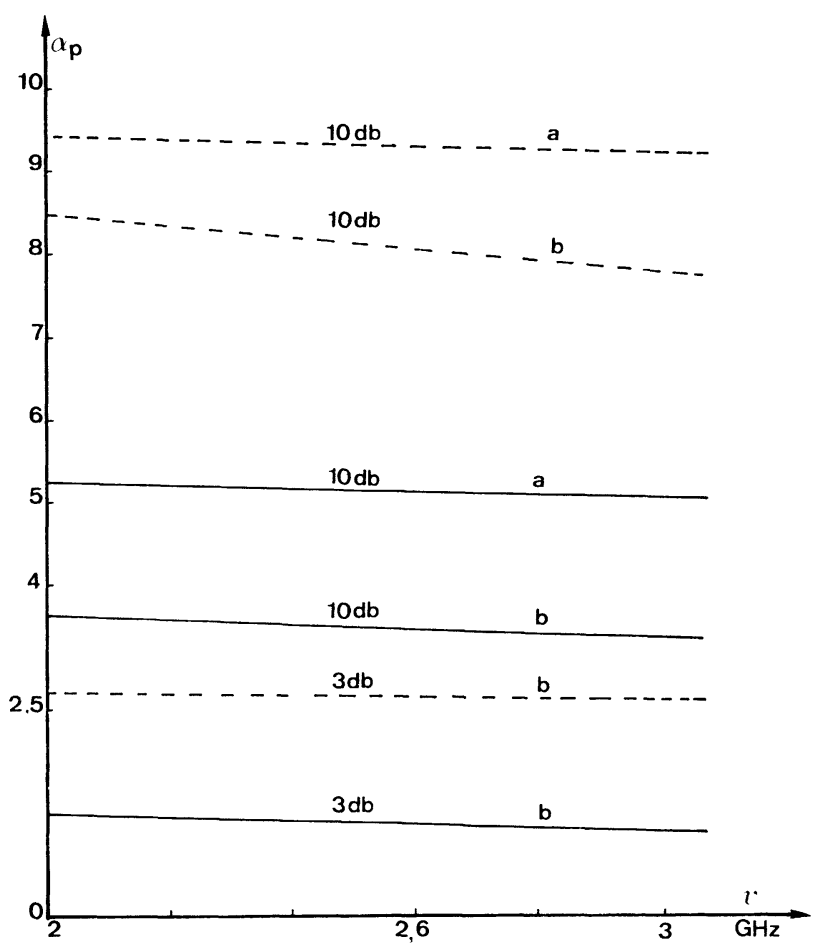

Fig. 4. - Courbes représentant les résultats expérimentaux du gain de puissance dans la boucle de recirculation : $a$ ) à onde stationnaire; $b$ ) à onde progressive. En pointillé, le cas des boucles courtes; en trait plein, le cas des boucles longues. 
tionnaire, la puissance rétrograde a été mesurée ; le coefficient de réflexion obtenu dans le cas de l'onde progressive est toujours resté inférieur à 0,03 ; dans le cas d'une onde stationnaire sa valeur varie entre 0,45 et 0,65 .

En comparant les résultats de la figure 4, on peut faire les remarques suivantes :

1) Dans le cas de la boucle courte, on se rapproche du couplage optimum; par contre, dans la boucle longue, on reste toujours en dessous de ce couplage. Il serait nécessaire dans ce cas de disposer d'un coupleur ajustable.

2) Lorsque la fréquence augmente, le coefficient $\alpha_{p}$ diminue, en raison de l'augmentation des pertes dans la boucle qui varient proportionnellement à la racine carrée de la fréquence [6].

3) Le coefficient de renforcement de la puissance dans l'anneau résonnant en régime d'onde stationnaire est plus important que celui obtenu dans l'anneau résonnant en régime d'onde progressive. En conséquence, pour une même puissance incidente, l'amplitude du champ déflecteur utile créé dans une ligne à méandres placée dans une boucle à onde stationnaire est supérieure à celle obtenue lorsque la ligne est située dans une boucle à onde progressive.

On peut donc s'attendre à une meilleure efficacité du déflecteur dans le cas d'une onde stationnaire.

5. Obtention de paquets courts. - Pour vérifier expérimentalement les propriétés du déflecteur à méandres placé dans un anneau résonnant, nous avons utilisé le dispositif suivant : un canon à électrons de type triode à cathode en borure de lanthane, suivi d'une lentille électrostatique à trois électrodes, fournit un faisceau fin d'électrons accélérés sous $30 \mathrm{kV}$ et permet d'obtenir à $1 \mathrm{~m}$ du canon, un spot de $1 \mathrm{~mm}$ de diamètre pour un courant de $1 \mathrm{~mA}$.

Le faisceau traverse, après la lentille, le déflecteur à méandres croisés placé dans un anneau résonnant, et est partiellement intercepté par un écran percé sur l'axe d'un trou de diamètre $d \simeq 1 \mathrm{~mm}$, situé à $50 \mathrm{~cm}$ de la sortie du déflecteur. Un collecteur, muni d'un diaphragme polarisé permet de mesurer le courant $I \mathrm{du}$ faisceau ayant traversé l'orifice en évitant toute émission secondaire (Fig. 5).

L'alignement $\mathrm{du}$ faisceau dans l'enceinte, où règne un vide de $10^{-6}$ torr, est rendu possible par l'emploi de quatre bobinages magnétiques rectangulaires qui annulent le champ magnétique terrestre sur l'axe du système.

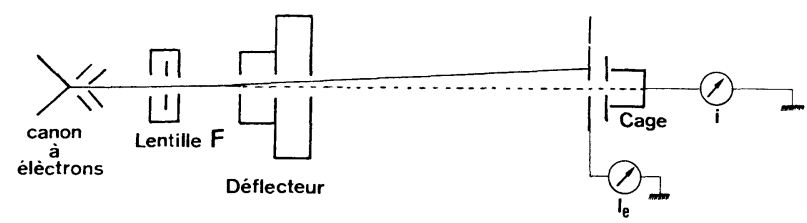

FIG. 5. - Schéma représentant le principe des mesures (indirectes) des paquets $\Delta \varphi_{0}$.
Ces bobines servent aussi à dévier légèrement le faisceau, de manière à amener la trace elliptique du faisceau à traverser le trou de l'écran situé sur l'axe optique.

L'alimentation du déflecteur provient d'un générateur à klystron reflex suivi d'un amplificateur à tube à onde progressive d'une puissance maximum $P_{\max }=10 \mathrm{~W}$ à $3 \mathrm{GHz}$.

Les mesures de courants $I_{\mathrm{E}}$ et $I_{0}$ ( $I_{\mathrm{E}}$ étant le courant intercepté par l'écran et $I_{0}$ le courant moyen reçu sur le collecteur) permettent d'estimer la largeur en phase $\Delta \varphi_{0}$ de chaque paquet. On a en effet :

$$
\Delta \varphi_{0}(\text { degrés })=\frac{360 I_{0}}{I_{\mathrm{E}}+I_{0}} .
$$

On en déduit la durée $\Delta \tau$ des impulsions de courant :

$$
\Delta \tau=\frac{1}{v} \cdot \frac{\Delta \varphi_{0}}{360} .
$$

On obtient pour $P_{\mathrm{i}}=8 \mathrm{~W}, \varepsilon_{\text {cin. }}=30 \mathrm{keV}, v=3 \mathrm{GHz}$, $d=1,5 \mathrm{~mm}$ et $I=1 \mathrm{~mA}$ :

Ligne dans un an-
$\begin{aligned} & I_{0}(\mu \mathrm{A}) \\ & \text { neau à onde progres- }\end{aligned}$
$\begin{aligned} & \text { sive } \\ & \text { Ligne dans un an- }\end{aligned}$
$\begin{aligned} & \text { neau à onde station- } \\ & \text { naire }\end{aligned}$

Par comparaison, l'utilisation du déflecteur à onde progressive utilisé sans anneau de recirculation, ne permet pas de produire des paquets de largeur inférieure à $\Delta \varphi_{0}=22^{\circ}\left(I_{0}=60 \mu \mathrm{A}\right)$ pour une même puissance injectée de $8 \mathrm{~W}$.

On peut visualiser aisément la trace elliptique obtenue par la déflexion du faisceau en plaçant un écran fluorescent au niveau de la cible (l'intensité du courant a été réduite à $10 \mu \mathrm{A}$ pour éviter la destruction de

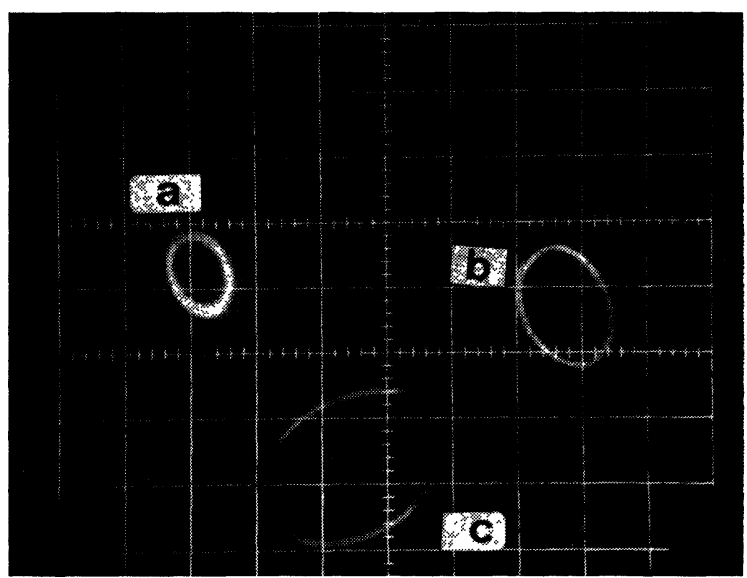

Fig. 6. - Ellipses obtenues sur un écran fluorescent par les trois méthodes de déflexion $: a$ ) en l'absence de boucle; $b$ ) avec une boucle à onde progressive ; $c$ ) avec une boucle à onde stationnaire. 
l'écran et la puissance incidente utilisée a été fixée à $3 \mathrm{~W}$ ).

La figure 6 donne pour comparaison les photographies des ellipses obtenues par les trois méthodes de déflexion: $a$ ) en l'absence de boucle; $b$ ) avec une boucle à onde progressive ; $c$ ) avec une boucle à onde stationnaire.

6. Conclusion. - L'utilisation d'un déflecteur à méandres placé dans une boucle de recirculation de la puissance $\mathrm{HF}$ et résonnant en onde stationnaire permet d'obtenir de meilleurs résultats que lorsqu'il est placé dans une boucle résonnant en onde progressive.

En effet, l'anneau à onde stationnaire permet de se rapprocher du couplage optimum et d'obtenir un renforcement maximum de la puissance circulant dans la boucle $(\simeq 9$ pour un coupleur de $10 \mathrm{~dB})$. Il permet ainsi d'obtenir des paquets d'électrons de faible extension en phase $\left(\Delta \varphi_{0} \simeq 8^{\circ}\right.$ avec une puissance HF injectée dans la boucle de $8 \mathrm{~W}$ en bande $\mathrm{S}$ ).

\section{Bibliographie}

[1] Boussoukaya, M. et SePtier, A., Nucl. Instrum. Methods 80 (1970) 109.

[2] BoussoukayA, M., Thèse de Doctorat ès-Sciences, I. E. F., Orsay (1975) p. 66.

[3] Bergeret, H., C. R. Hebd. Séan. Acad. Sci. 268 (1969) 733.
[4] Boussoukaya, M. et SePtier, A., Nucl. Instrum. Methods 117 (1974) 321.

[5] Moreira, A., Ann. Radioélectr. 15 (1961) 230.

[6] Loew, G. A. et NeAL, R. B., Linear Accelerators (ed. North Holland, Amsterdam) 1970, p. 39.

[7] SePtier, A., Vide 118 (1965) 328. 